\title{
Multidimensional scaling of color difference in the pigeon*
}

\author{
BRUCE SCHNEIDER \\ Columbia University, New York, New York 10027
}

Pigeons were required to discriminate between "identical" vs "different" pairs of lights in a yes/no signal-detection task with a symmetrical payoff matrix. If the two lights projected on the two halves of the bipartite field constituting the center response key in a three-key chamber were identical in wavelength composition, a single peck on the right key was reinforced with food. If the two lights differed in wavelength composition, then left-key pecks were reinforced. In Experiment 1, each of six pigeons experienced all possible pairs of 11 spectral lights and 1 purple light (66 pairs). In Experiment 2, the set of lights was expanded to 15 (105 pairs) and included a white light. The percentage of correct choices was taken as an index of the dissimilarity between the two lights constituting a pair. The rank-order information available in these dissimilarity measures was used to determine coordinate projections for each light in a two-dimensional Euclidean space. The configuration obtained in this manner was interpreted as a color circle for the pigeon.

Certain aspects of the phenomenological appearance of colors suggest that they are best represented geometrically by a circular configuration (the ubiquitous color circle or wheel). Long and short wavelength lights (reds and violets) appear perceptually similar; and, in fact, a graded series of colors (the purples) can be constructed between them by using the appropriate mixtures of a long and $a$ short wavelength light. Thus, one can proceed in imperceptibly small steps through violet, blue, green, yellow, red, purple, and back to violet again. In a geometric representation, the similarity of two colors is indicated by their proximity. Hence, in order to represent the perceptual similarity in this closed series, the spectrals and purples are arranged in a circular configuration.

In addition, the center of the circle is usually chosen to represent the achromatic experience, i.e., the experience associated with a white light. In this way, the saturation of a light can be represented in terms of a radial spoke from the center of the circle to its perimeter. Various degrees of saturation of a color of a specific hue would be represented by various points along a spoke connecting the achromatic center to the locus of the identical, fully saturated, hue on the circle's perimeter.

A color circle, incorporating these

*I would like to thank Dr. Donald Hood. Dr. J. A. Nevin, Mr. Scott Paxkr, and Dr. A. A. Writht for their helpful comments and surgentions. I would also like to thank Mr. Martin Thompion and Mr. Dudley Anderson for their unfalling andistance. This research was supported, in part, by I.S.R.P. funds from Columbia Univerity. Reprints may be obtained from B. Schineider, Department of Psycholosy, Columbia Univeratty, New Yot, New Yotk 10027. features, is quite useful since it represents, at a glance, the similarity among colors. However, a problem still remains with respect to an appropriate interpretation of the distance between any two colors. If a geometric representation is to have validity, the distance between any two points should have some psychological (experiential) counterpart. The most obvious psychological interpretation of the distance between two colors in this geometric representation is their perceived dissimilarity.

Hering (see translation by Hurvich \& Jameson, 1964) was the first to point out the desirability of having the distance between any two colors in a geometric representation such as the color circle directly represent their psychological dissimilarity. In a section discussing color differences, Hering said: "In an ideal color series or an ideal color line ... the separation of two colors would be a measure both of their difference and of their similarity, for the magnitude of their difference would be directly proportional and the magnitude of their similarity inversely proportional to their separation; color pairs whose individual members are equally different from each other, or equally different color pairs ... would have their component colors separated by the same distance in the color series; twice the distance in any part of the series would correspond to a color difference that is twice as great, and so on [Hering, 1964, p. 36 ]." Hering did not pursue the quantification of color difference, and indeed at that time, techniques did not exist for such quantification. Nevertheless, he proposed that distances between colors in a geometric representation should directily reflect the magnitude of their psychological dissimilarity.
The present experiments are an attempt to establish a geometric representation for color experience in the pigeon, in which the distance between points in the space represents the extent of the "perceived dissimilarity" of the colors associated with these points. To establish such a geometric representation, it is necessary: (1) to obtain measures from pigeons of the psychological dissimilarity between colors, i.e. measures of color difference, and (2) to use these measures of color difference to determine coordinate projections for the stimuli in a two-dimensional color space.

In Experiment 1, measures of the dissimilarity of pairs of spectral lights and a purple light were obtained from the pigeon using a yes/no signal-detection procedure. Pigeons were required to discriminate between "identical" vs "different" pairs of lights. The accuracy of this discrimination with respect to a given pair of lights was taken as a measure of the color difference between them. These accuracy measures were used to determine coordinate projections for the stimuli in a two-dimensional Euclidean space. Shepard (1962) has shown that measures of the distance between stimuli in a spatial representation need not be directly proportional to the actual distance between them in order to determine the coordinate projections of the stimuli. All that is required is that the dissimilarity measures be monotonically related to the distance between stimuli. Thus, the rank-order information in the dissimilarity measures is sufficient to determine a spatial embedding (determine the coordinate projections) for a set of colors.

In Experiment 2, additional monochromatic lights and a white light were included in the stimulus set to determine the location of the achromatic experience in this two-dimensional representation.

\section{METHOD}

Subjects

The Ss were six white Carneaux pigeons from the Palmetto Pigeon Plant, Sumpter, South Carolina. With the exception of the first $\mathbf{4 0}$ sessions of Experiment 2, they were tested 7 days per week and fed enough grain after each session to maintain them at $85 \%$ of their ad lib weights. During the first 40 sessions of Experiment 2, they were not tested on the weekends. In addition, a vitamin supplement was added to their water once a week.

\section{Apparatus}

(1) Experimental chamber. The experimental chamber was a modified 


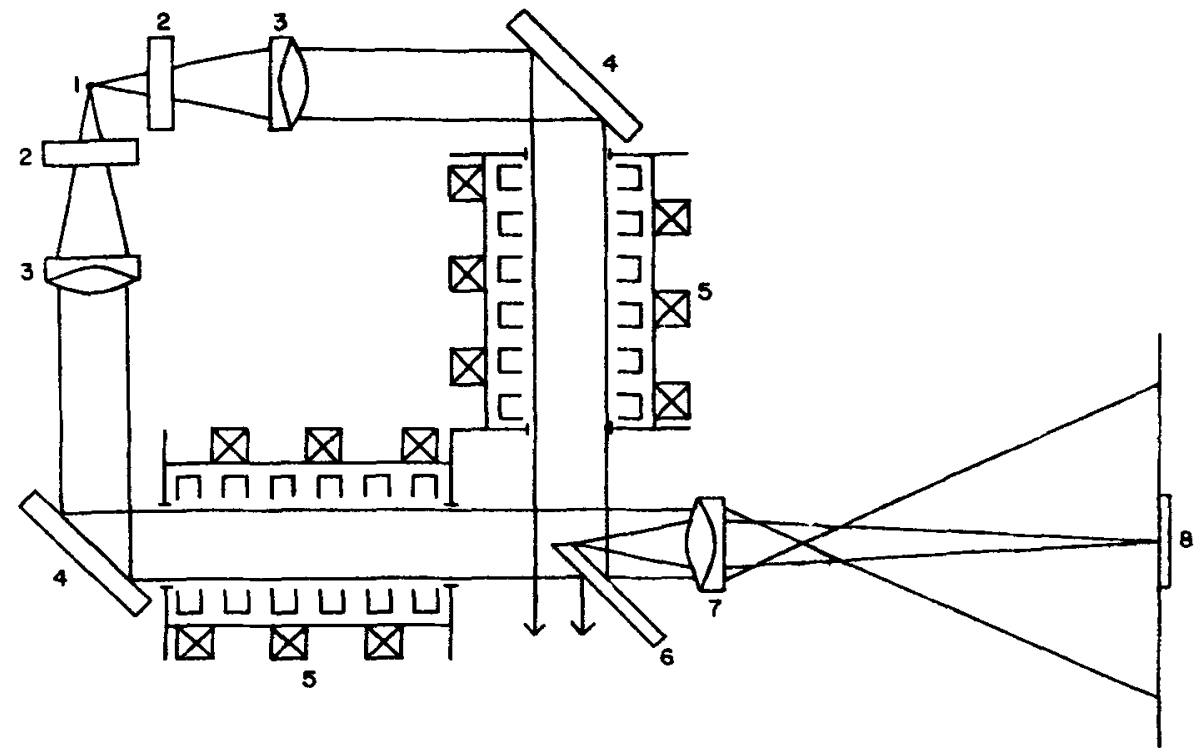

Fig. 1. Schematic of the optical system employed in Experiments 1 and 2.

ice chest. An aluminum panel separated the Ss' compartment (24.1 $\mathrm{x}$ $27.9 \times 33.0 \mathrm{~cm}$ ) from the stimulus and food delivery devices. Three Lehigh Valley response keys were centered behind three $2.5-\mathrm{cm}$ holes in this pand. All three response keys were positioned $24.1 \mathrm{~cm}$ above the wire-mesh grid that served as the floor of the chamber. The middle response key was centered on the panel, and the two side-keys were displaced $7.6 \mathrm{~cm}$ from the center of the panel. Each side key could be illuminated by a G.E. $182928-\mathrm{V}$ bulb. The intensity of the bulb was reduced by a 75-ohm series resistor. The luminance of the side key was $14 \mathrm{~mL}$.

Located $16.5 \mathrm{~cm}$ below the center key was a $6.35 \times 5.1 \mathrm{~cm}$ opening in the panel through which a hopper filled with mixed grain could be made available. The reinforcer was $4.0 \mathrm{sec}$ access to grain. During the reinforcement cycle, the hopper was illuminated by a Sylvania 24 ESB bulb. White noise was continuously presented.

Four G.E. 1829 28-V bulbs, mounted behind a $12.7 \times 16.5 \mathrm{~cm}$ piece of white plastic on the ceiling of the chamber were used to maintain a constant state of adaptation. The average luminance of the chamber walls as measured by an Iford S.E.I. photometer was $0.27 \mathrm{~mL}$. The adaptation light remained on for the entire session except for the occasional 4-sec blackout periods which followed incorrect choices.

(2) Optical system. A bipartite field (2.5-cm diam) was projected directly on the center key by the modified projection system shown in Fig. 1. The light source (1) was a voltage-regulated 1,000-W Tungsten-Halogen bulb. The spectral emission between 420 and $600 \mathrm{~nm}$ was best fitted by a black body radiator with a color temperature of $3,000^{\circ} \mathrm{K}$. Ultraviolet and infrared filters attenuated the energy at longer and shorter wavelengths. Two beams of light were taken from the source to form the separate halves of the bipartite stimulus. In each channel, light from the source passed through heat-absorbing glass (2) and a collimating lens (3). After being reflected from a front surface mirror (4), the collimated light passed through a filter box (5) containing interference, neutral density, and blocking filters. The two channels were then united by a front-surface mirror (6). A condensing lens (7) focused the edge of the mirror on the ground-glass screen that constituted the center response key. The width of the slit separating the two halves of the field was $0.3 \mathrm{~mm}$.

Solenoid devices in the filter boxes interposed the interference, neutral density, and blocking filters programmed for a particular trial. The filter boxes were mounted independently of the rest of the optical system to minimize vibration.

The wavelength of the stimuli was controlled by Bausch and Lomb No. 44 interference filters, which pass three modes of energy: one in the ultraviolet, one in the visible, and one in the infrared. The ultraviolet mode was eliminated by blocking filters. The wavelength of the ultraviolet mode determined the blocking filter employed (Kodak Wratten $2 \mathrm{~A}$ or 15 filter). The heat absorbing glasses eliminated the infrared mode of energy. The intensity of the visible mode was controlled by Kodak
Wratten neutral density filters.

(3) Stimulus calibration. In calibrating each half of the bipartite field, the mirror uniting the two channels of the optical system was positioned so that light from only one of the channels was incident on the ground-glass response key. To determine peak wavelength, an Edgerton, Germeshausen, and Grier 580-585 spectroradiometer was positioned in front of the response key. As Tables 1 and 2 show, the peak wavelengths for nominally matched filters for the two channels do not always coincide. The maximum deviation is $4 \mathrm{~nm}$ (Stimuli No. 14). In these experiments, the two halves of the field were considered identical in wavelength if they were produced by the two filters comprising a matched pair (same stimulus number). The two halves of the field were considered as differing in wavelength if they were produced by two filters which were not from a matched pair. The nominal wavelength value for a matched pair is the mean of the two peak wavelengths.

The relative radiance of each half of the bipartite field was determined by positioning the radiometer in front of the ground-glass key. The intensity of the stimuli was adjusted by adding Kodak Wratten neutral density filters so that the lights were photometrically equated to the 552-nm stimulus, using luminosity coefficients obtained by Blough (1957) for pigeons. Tables 1 and 2 indicate the accuracy of the photometric matches. Note that Stimuli 1 and 2 show the greatest departure from equality $(-0.7$ and $-0.3 \log$ unit, respectively). To have attempted to equate all wavelengths photometrically in the present apparatus would have reduced the luminance of the monochromatic lights to less than optimum photopic levels. Consequently, it was decided that these two stimuli would be included so that a broad spectrum could be investigated even though they were not photometrically equated. The importance of their departure from photometric equality was assessed in a control experiment.

A blocking filter was not employed for the interference filter with peak wavelength of $670 \mathrm{~nm}$ (No. 14). Consequently, the shorter mode was also present $(454 \mathrm{~nm})$. Such a combination of a long and short wavelength defines a human purple. This filter was photometrically equated as follows. The lower peak was first blocked and a radiometer reading obtained. Second, a radiometer reading was obtained without the blocking filter. The difference in the two readings was taken as the measure of the energy in 
Table 1

Stimuli Employed in Experiments 1 and 2: Channel 1

\begin{tabular}{|c|c|c|}
\hline Stimulus & $\begin{array}{c}\text { Wavelength } \\
\text { Composition } \\
\text { (in Nanometers) }\end{array}$ & $\begin{array}{l}\text { Difference from } \\
\text { Photometric Equality } \\
\text { With Stimulus } 7 \\
\text { (in Log Units) }\end{array}$ \\
\hline $\mathbf{1}$ & 454 & -0.70 \\
\hline $\mathbf{2}$ & 472 & -0.33 \\
\hline 3 & 491 & -0.02 \\
\hline 4 & 510 & -0.03 \\
\hline 5 & 529 & +0.02 \\
\hline 6 & 542 & -0.01 \\
\hline 7 & 552 & 0.00 \\
\hline 8 & 569 & -0.03 \\
\hline 9 & 592 & -0.05 \\
\hline 10 & 599 & +0.01 \\
\hline 11 & 610 & -0.05 \\
\hline $\mathbf{1 2}$ & 633 & +0.07 \\
\hline 13 & 653 & -0.02 \\
\hline 14 & 453 and 668 & -0.10 \\
\hline 15 & White Light & +0.07 \\
\hline
\end{tabular}

Table 2

Stimuli Employed in Experiments 1 and 2: Channel 2

\begin{tabular}{ccc} 
Stimulus & $\begin{array}{c}\text { Wavelength } \\
\text { Composition } \\
\text { (in Nanometers) }\end{array}$ & $\begin{array}{c}\text { Difference from } \\
\text { Photometric Equality } \\
\text { With Stimulus 7 } \\
\text { (in Log Units) }\end{array}$ \\
\hline 1 & 457 & -0.69 \\
2 & 473 & -0.30 \\
3 & 490 & +0.30 \\
4 & 512 & -0.02 \\
5 & 527 & +0.03 \\
6 & 542 & -0.01 \\
7 & 552 & 0.00 \\
8 & 567 & +0.02 \\
9 & 589 & -0.05 \\
10 & 589 & -0.04 \\
11 & 608 & -0.02 \\
12 & 630 & +0.06 \\
13 & 653 & -0.01 \\
14 & 456 and 672 & 0.00 \\
15 & White Light & +0.07 \\
\hline
\end{tabular}

the higher peak. The two peaks were separately corrected for the spectral response of the radiometer and the pigeon. The two corrected readings were then summed. Finally, neutral density filters were added until the sum of the corrected readings was identical to the corrected reading for the 552-nm stimulus.

The stimulus labeled "white" in Tables 1 and 2 was produced by interposing only neutral density filters in the collimated beam. This stimulus was equated photometrically by first positioning the spectroradiometer in front of the key. Second, readings were determined for 5-nm bands from 415 to $700 \mathrm{~nm}$. Third, these readings were adjusted for the spectral sensitivity of the radiometer, the pigeon, and the relative absorption of Kodak Wratten filters. These corrected readings were plotted as a function of wavelength, and the area under the curve was determined. This curve defines "pigeon luminance" (arbitrary unit). Pigeon luminance was determined for the 511-nm stimulus in the same way. The log unit difference in luminance between the 511-nm stimulus and the white light was then computed, and the white light source was attenuated the number of $\log$ units specified in the calculation above.

The luminance of the $580-\mathrm{nm}$ stimulus was $30 \mathrm{~mL}$, as determined by an Ilford SEI photometer.

\section{Procedure}

(1) Final procedure. At the beginning of a trial, the bipartite field was projected on the center key. The side keys were dark. A single response on the center key (an "observing" response) illuminated the side keys. If the two halves of the center key were identical in wavelength, then a response to the right-side key was designated as correct. If the two halves of the field differed in wavelength, then a response on the left-side key was designated as correct. Four-second access to food followed $20 \%$ of the correct (right or left) responses. The other $80 \%$ were followed by $0.25 \mathrm{sec}$ access to food. This latter time period is too brief for the pigeon to be able to eat any grain. It simply served as "feedback" for correct responses. Incorrect responses (responding left for identical wavelengths and right for different wavelengths) produced $4 \mathrm{sec}$ of blackout. During a blackout, all lights, including the overhead adaptation light, were extinguished. Time between a side key response and the beginning of the next trial was 14.0 sec. Keys were extinguished when a response occurred to a lighted side key. Each session consisted of 192 trials.

The procedure is equivalent to a yes/no signal-detection paradigm with a symmetrical payoff matrix. Only a single pair of stimuli was employed per daily session. The four possible stimulus combinations appeared during a session with equal frequency: (1) Stimulus $i$ on both halves of the field; (2) Stimulus $j$ on both halves of the field; (3) Stimulus $i$ on the left and Stimulus $j$ on the right; and (4) Stimulus $j$ on the right and Stimulus $i$ on the left. Since the payoff matrix was constant throughout Experiments 1 and 2, response bias should remain constant. Thus, the percentage of correct responses should be monotonically related to $\mathrm{d}^{\prime}$ or other measures of discriminability.

In Experiment 1, each pigeon experienced all possible pairs (66) of 12 stimuli $(1,2,3,4,5,7,8,9,10$, $11,13,14)$. Only one pair was presented per daily session. Each bird was presented these 66 pairs in a different random order. At the end of the 66-day sequence, 5 stimuli chosen to span the range from near-chance level responding to near-perfect discrimination were presented again to the six birds to check whether the accuracy of discrimination improved over the 66 sessions.

In Experiment 2, the stimulus set was expanded to include all of the 15 stimuli listed in Tables 1 and 2. Thus, each bird was presented with the 105 possible pairs of stimuli, one pair per day. At the end of the 105 sessions, 6 stimuli, chosen to span the range from near-chance level responding to near-perfect discrimination, were presented again to check whether the accuracy of discrimination improved over the 105 sessions. Finally, to see if lack of photometric equality for Stimuli 1 and 2 affected the accuracy of discrimination, Stimulus 1 was photometrically equated with Stimulus 4 and presented during a session. Stimulus 2 was similarly paired with a photometrically equal Stimulus 3 and presented during a session.

\section{Preliminary Training}

After being trained to peck the center and side keys in seque ce for food reinforcement, each of 12 is was directly introduced into the signal-detection task described above. The only differences were that correct responses were reinforced on $100 \%$ of the trials and only 96 trials were programmed per daily session. Furthermore, the same stimulus pair $(5,9)$ appeared each day. After 50 sessions, the number of trials was increased to 192 and reinforcement for correct responses was reduced from $100 \%$ to $20 \%$. Three birds were dropped from the experiment after 40 days, and three more were eliminated after 75 days. The remaining six birds were selected for further study because of their high degree of accuracy in performing this task.

During the first 75 days of exposure 
to the signal-detection task, only Filters 5 and 9 were presented. By this time, the percentage of correct responses had stabilized at $95 \%$ (averaged over six birds and the 71-75 sessions). The Ss were then presented other pairs of stimuli until the percentage of correct responses appeared stable for these pairs. Some of the pairs required relatively easy and some relatively difficult discriminations. This phase took 53 sessions.

After 128 sessions of exposure to this task, all possible pairs of 11 stimuli ( 55 pairs) were presented to each $S$, one pair per day. The stimuli were Numbers $1,2,3,4,5,7,8,9,11$, 13 , and 14 from Tables 1 and 2 . (Stimulus 14 at this time had the lower peak blocked and therefore contained energy only at the 670 mode.) However, since they were not at this time equated for brightness for the pigeon, the data from this series were not used to construct a color circle. At the end of this series, the stimuli were equated for brightness and after 15 sessions of exposure to random pairs of these newly calibrated stimuli, Experiment 1 was begun.

Infrequently, a filter would become stuck during a session. In that event, the data for that bird on that day were deleted and the stimulus pair repeated at the end of the experiment. Such failures were quite rare.

\section{RESULTS}

In Experiment 1, each of six pigeons experienced every possible pair of 12 stimuli (66 pairs). The accuracy of discrimination for a pair (number of correct choices divided by number of trials) was taken as a measure of the dissimilarity of the two stimuli comprising that pair. A rank was then assigned to each of the 66 stimulus pairs based on these accuracy measures. These ranks represent an ordinal scale of perceived color difference for the pigeon. Kendall's coefficient of concordance (W) was used to determine the extent of the agreement among the rankings for the six pigeons. The coefficient of concordance was 0.68 in Experiment 1, indicating relatively good agreement across Ss.

These ranks were then averaged across Ss to obtain a rank ordering of color differences. A two-dimensional spatial configuration was determined from the average ranks, using the iterative technique described by Carvellas and Schneider (1972). The loci of the stimuli in this two-dimensional Euclidean space are represented by the squares in Fig. 2 .

Also shown in Fig. 2 is the spatial configuration determined from the rank order of perceived color

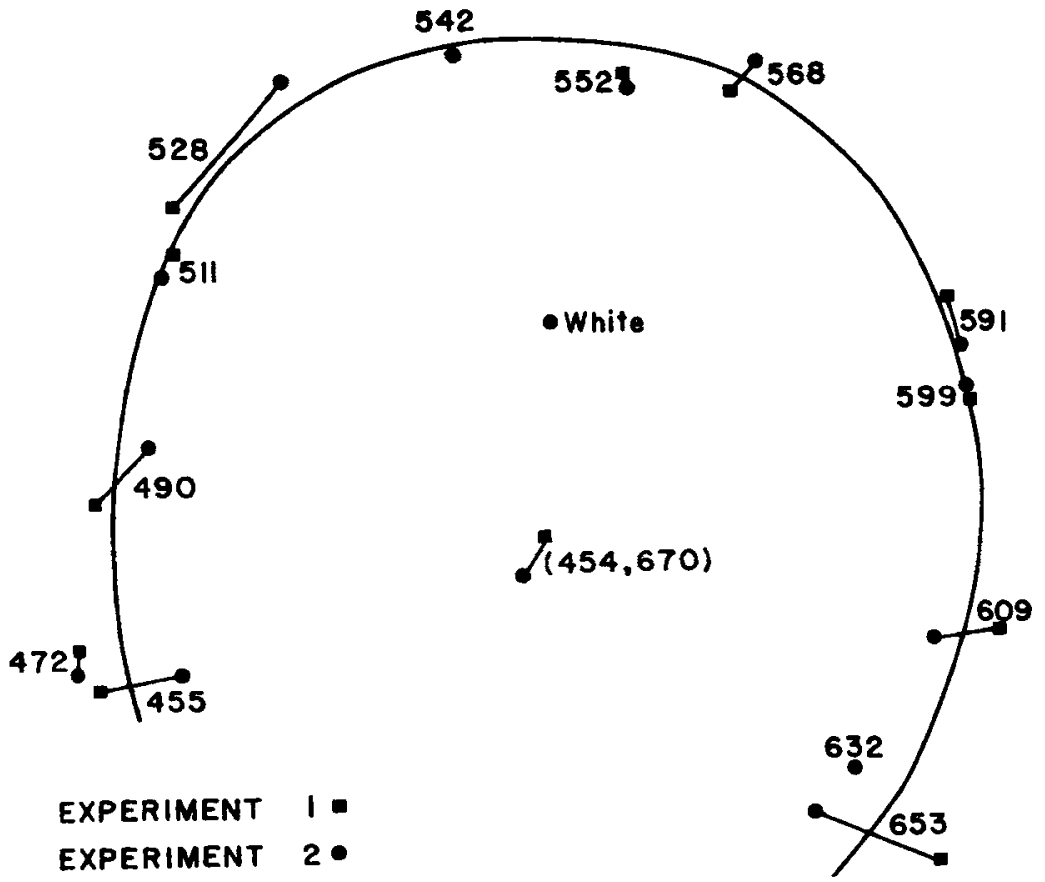

Fig. 2. A two-dimensional color space for the pigeon.

difference for the 105 stimulus pairs of Experiment 2. As in Experiment 1, a rank was assigned to each stimulus pair for each pigeon based on the accuracy with which the stimuli comprising a pair were discriminated. The coefficient of concordance (W) among the ranks was 0.70 , again indicating a relatively good agreement in the ranking of stimulus pairs across pigeons. These ranks were then averaged, and the average ranks used to determine the loci of the stimuli (circles) in the two-dimensional Euclidean space shown in Fig. 2.

Figure 2 shows that stimuli which were common to Experiments 1 and 2 occupy approximately the same loci in the two-dimensional configuration. A smooth line, drawn through the loci of the monochromatic lights, indicates that the monochromatic lights are arranged in a roughly circular pattern, with the long and short wavelengths relatively close together. The locus of the white light source, rather than being equally distant from all monochromatic lights, is situated closest to $550 \mathrm{~nm}$ (a human yellow-green). The mixed wavelength light $(454+670$, a human purple) does not lie on an extension of the circular configuration connecting the long and short wavelengths, but is displaced toward the center of the configuration and is situated relatively close to the locus of the white light.

Goodness of fit of the two configurations was evaluated using Kruskal's (1964) stress measure where:

$$
\text { Stress }=\sqrt{\frac{\sum_{i j}\left(D_{i j}-\hat{D}_{i j}\right)^{2}}{\sum_{i j} D_{i j}^{2}}}
$$

The $D_{i j}$ in this formula are the predicted interpoint distances and the $\hat{D}_{\mathrm{ij}}$ are the best-fitting monotone distances to the obtained dissimilarities (see Kruskal, 1964). Kruskal's measure simply normalizes the sum of square differences between the predicted and best-fitting monotone distances, permitting a comparison of goodness of fit across experiments. Stress for the two configurations was $6 \%$ and $14 \%$ for Experiments 1 and 2, respectively. According to Kruskal's recommended scoring scheme, this represents a "good" and "fair" fit, respectively.

To determine whether discrimination accuracy increased over the course of Experiment 1, five stimulus pairs were repeated at the end of that experiment. Similarly, six stimulus pairs were repeated after Experiment 2. These pairs were, in each instance, chosen so that they covered a wide range in discrimination accuracy. Figure 3 shows the accuracy of discrimination for the replicated pairs as a function of the accuracy of the discrimination for the identical pairs in the experiment proper. The data points are an average over six pigeons, and the line with unit slope represents perfect replication. Figure 3 indicates that there might be a slight improvement in the accuracy of 
discrimination over the course of the experiment since 8 of the 11 replication points lie slightly above this line. However, the maximum displacement from the line never exceeds $5 \%$, indicating rather stable performance during Experiments 1 and 2.

Since Stimuli 1 and 2 were not completely equated photometrically, two additional sessions were conducted in which Stimulus 1 was photometrically equated with Stimulus 4, and Stimulus 2 was photometrically equated with Stimulus 3. When Stimuli 2 and 3 (suitably equated) were then presented to the pigeons, there was only a 1\% decrease in accuracy when compared to the nonequated condition. However, when Stimuli 1 and 4 were presented, discrimination accuracy decreased $7 \%$ when compared to the Stimulus 1 represented the greatest degree of departure from photometric equality (approximately $0.7 \mathrm{log}$ units), it may be that the luminance difference was contributing to the accuracy with which this stimulus was discriminated from the others. However, it is unlikely that luminance difference affected the accuracy with which Stimulus 2 was discriminated from the others.

\section{DISCUSSION} from Experiments 1 and 2 are intended as a geometric representation of perceived color difference in the pigeon. Three factors indicate the appropriateness of this interpretation nonequated condition. Since

The two configurations obtained

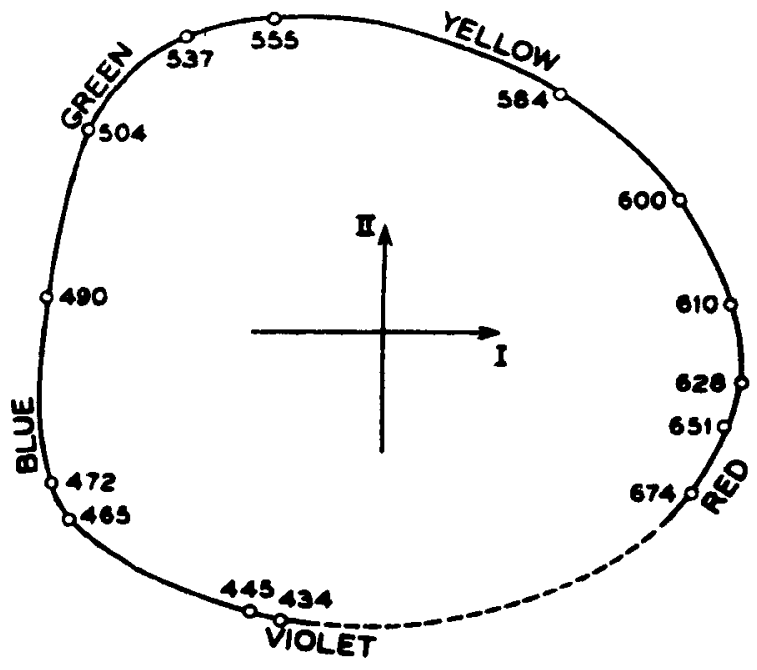

Fig. 4. A two-dimensional color space for humans. Data collected by Ekman (1954), analyzed by Shepard (1964).

of the dissimilarity measures. First, the two configurations in Fig. 2 exhibit substantial agreement, i.e., stimuli common to both experiments have approximately the same loci. Second, the stress values obtained in these experiments are reasonably low. A stress value of $0 \%$ indicates that the interpoint distances in the obtained configuration are, without exception, monotonically related to the dissimilarity measures, i.e., a Euclidean representation of the stimuli is perfectly consistent with the obtained rank-ordering of the dissimilarities. The stress values actually obtained in these experiments $(6 \%$ and $14 \%)$ suggest that a geometric interpretation

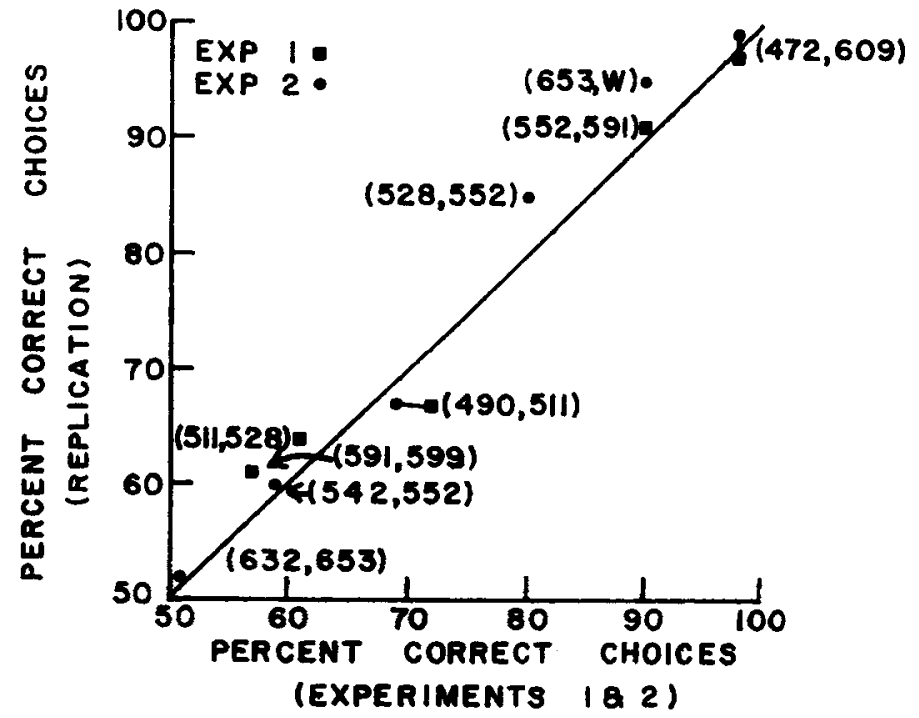

Fig. 3. Discrimination accuracy (average of six pigeons) in a replication as a function of discrimination accuracy in Experiments 1 and 2. Stimuli common to both replications are connected by straight lines. The line of unit slope represents perfect replication. is reasonable in the sense that the rank order of the interpoint distances in this representation agrees quite well with the rank order of the dissimilarity measures. Third, the configuration obtained in Fig. 2 provides a representation of hue that is similar to that obtained for humans. Figure 2 indicates that the monochromatic lights are best represented by a roughly circular configuration. Results for human observers yield similarly shaped configurations. Ekman (1954) had Ss rate the similarity of all possible pairs of 14 monochromatic lights. (He did not report whether these lights were equated for brightness.) Shepard (1964) took the rank order of these similarities and determined a spatial embedding for these stimuli. The results are shown in Fig. 4. Note that the configuration is roughly circular and that the long and short wavelengths are perceptually similar, i.e., appear close together in this spatial configuration.

\section{Comparisons with Other Studies with Pigeons}

With respect to wavelength similarity for the pigeon, some results of Wright and Cumming (1971) are in agreement with the suggestion in Fig. 2 that the long and short wavelengths are perceptually similar for the pigeon. Wright and Cumming (1971) trained their pigeons to peck the side key which was the same color as the center key in a three-key chamber. Thus, if the center key was illuminated by $600-\mathrm{nm}$ light, then a response to the similarly illuminated side key was reinforced. The other side key was illuminated by a light of a different wavelength. During a probe condition, $458 \mathrm{~nm}$ appeared on one side key and 650 on the other, when 
the center key was illuminated by a 600-nm light. Under these conditions, some birds pecked the shorter wavelength. This would be expected if the long and short wavelengths were perceptually similar.

The spacing of the wavelengths around the perimeter of the configuration in Fig. 2 is consistent with wavelength discrimination data collected by Wright (1972) for the pigeon. Wright found that the wavelength discrimination function contained minima at 500,540, and $600 \mathrm{~nm}$ (i.e., differential sensitivity was greatest at those wavelengths). The spacing in Fig. 2 between adjacent wavelengths is largest in these regions.

The spacing of monochromatic lights in Fig. 2 is also consistent with Shepard's (1965) analysis of Guttman and Kalish's (1956) and Blough's (1961) data. In these experiments, pigeons were trained to peck a disk illuminated with one value of monochromatic light. After several training sessions, generalization tests were conducted during which different wavelengths were projected on the disk and the number of responses to these lights was recorded. Responding was found to be maximal at the training stimulus and declined as the difference between training and test stimuli increased. Several different values of training stimuli were employed. Shepard (1965) transformed the wavelength scale so that all of these several generalization gradients were of the same shape. Spacing along this transformed scale was maximal at about 490,530 , and $610 \mathrm{~nm}$, indicating good agreement with both the present study and Wright's (1972) results.

\section{The Representation of Saturation}

In the human color circle, the achromatic experience is represented by a point in the center of the circle. The white light source in this experiment appears achromatic to a human observer, i.e., it is very unsaturated. If it also appears achromatic to the pigeon, its locus in Fig. 2 suggests a similar geometric representation for saturation in the pigeon. However, its location with respect to the monochromatic lights indicates that the spectral colors for the pigeon are not equally saturated. The distance between the perimeter of the circle and the locus of the white light is minimal at about $550 \mathrm{~nm}$, suggesting that this wavelength defines the least saturated spectral color for the pigeon.

The mixed wavelength light (454, $670)$, although it lies between the long and short wavelengths, is situated relatively close to the white light. This suggests that it appears relatively desaturated to the pigeon. Since this stimulus contains energy at two modes in the visible spectrum, it is not surprising that its saturation is less than that of a monochromatic light. Thus, there is reason to suspect that lights of colorimetric purity less than 1.0 might be situated on a line connecting the white light source to monochromatic light with the same dominant wavelength.

\section{Theoretical Implications}

Shepard (1966) has shown that the rank-order information in a set of interstimulus comparisons (number of stimuli $\geqslant 10$ ) is sufficient to determine a configuration in a Euclidean space that is unique up to a linear translation, rotation, or reflection. Distances between points in such a configuration constitute measurement on a ratio scale. Therefore, the distances between stimuli in Fig. 2 measure the extent of perceived color difference on a ratio scale. A psychological interpretation of this configuration, with saturation being represented by the distance from the achromatic light and with hue corresponding to radial "spokes" from the achromatic "center," is consistent with other psychophysical studies employing pigeons as Ss, and with respect to the manner in which saturation varies as a function of colorimetric purity for human Ss.

A problem still remains, however, with respect to the interpretation of the coordinate axes of this "psychological" space. Is there a rotation which would yield a meaningful set of coordinate axes, i.e., axes which were consistent with known or theoretical mechanisms of color vision? Hering (see translation by Hurvich \& Jameson, 1964), in his discussion of color perception, argued for a meaningful psychological interpretation. He proposed that chromatic color experiences could be described as the operation of two opponent processes, a red-green process and a blue-yellow process. These two processes, according to Hering, could be represented as orthogonal dimensions in a two-dimensional space, with the origin representing equal stimulation of each opponent process. This equal balancing of two factors would lead to the experience of white light. The modern version of this theory is elaborated by Hurvich and Jameson (1957).

It is also clear from Hering's discussion of color difference that he wished to interpret distances between colors as specifying psychological dissimilarity (see introduction). Presumably, the present configuration incorporates this feature, namely that distances between stimuli in the color space be directly proportional to their psychological dissimilarity. Furthermore, two dimensions appear sufficient in the sense that stress is low for both experiments. However, it is not possible to identify a psychologically meaningful set of coordinate axes in Fig. 2 . Such a psychological (or physiological) interpretation, if appropriate, awaits the demonstration of chromatic opponent processes in the pigeon and proper identification of the peak wavelengths associated with the opponent processes.

\section{REFERENCES}

BLOUGH, D. S. Spectral sensitivity in the pigeon. Journal of the Optical Society of America, 1957, 47, 827-833.

BLOUGH, $D$. $S$. The shape of some wavelength generalization gradients. Journal of the Experimental Analysis of Behavior, 1961, 4, 31-40.

CARVELLAS, T., \& SCHNEIDER, B Direct estimation of multidimensional tonal dissimilarity. Journal of the Acoustical Society of America, 1972, 51, 1839-1848.

EKMAN, G. Dimensions of color vision. Journal of Psychology, 1954, 38, 467-474.

GUTTMAN, N., \& KALISH, H. I Discriminability and stimulus generalization. Journal of Experimental Psychology, 1956,51, 79-88.

HERING, E. Outlines of a theory of the light sense. Trans. L. M. Hurvich and D. Jameson. Cambridge: Harvard Univergity Press, 1964.

HURVICH, L. M., \& JAMESON, D. An opponent-process theory of color vision. Psychological Review, 1957, 64, 384-404. KRUSKAL, J. B. Multidimensional scaling by optimizing goodness of fit to a nonmetric hypothesis. Psychometrika, $1964,29,1-28$.

SHEPARD, $R$, N. The analysis of proximities: Multidimensional scaling with an unknown distance function. I Psychometrika, 1962, 27, 126-140.

SHEPARD, $R$. N. Extracting latent structure from behavioral data. In Proceedings of the 1964 sympostum on digital computing. Bell Telephone Laboratories, May 1964.

SHEPARD, R. N. Approximation to uniform gradients of generalization by monotone transformation of scale. In $D$. I. Mostofsky (Ed.), Stimulus generalization. Stanford: Stanford University Press, 1965. Pp. 94-111.

SHEPARD, R. N. Metric structures in ordinal data. Joumal of Mathematical Psychology, 1966, 3, 287-315.

WRIGHT, A. A. Psychometric and psychophysical hue discrimination functions for the pigeon. Vision Research, in press.

WRIGHT, A. A., \& CUMMING, w. W. Color-naming functions for the pigeon. Journal of the Experimental Analysis of Behavior, 1971, 15, 7-17.

(Received for publication June 1, 1972 accepted for publication July 7, 1972.) 